\title{
Studying neutrinos at the LHC: FASER and its impact to the cosmic-ray physics
}

\author{
Akitaka Ariga ${ }^{a, b, *}$ on behalf of the FASER Collaboration \\ (a complete list of authors can be found at the end of the proceedings) \\ ${ }^{a}$ Albert Einstein Center for Fundamental Physics, Laboratory for High Energy Physics, University of Bern, \\ Sidlerstrasse 5, CH-3012 Bern, Switzerland \\ ${ }^{b}$ Department of Physics, Chiba University, 1-33 Yayoi-cho Inage-ku, Chiba, 263-8522, Japan \\ E-mail: akitaka.ariga@lhep.unibe.ch
}

\begin{abstract}
Studies of high energy proton interactions have been basic inputs to understand the cosmic-ray spectra observed on the earth. Yet, the experimental knowledge with controlled beams has been limited. In fact, uncertainties of the forward hadron production are very large due to the lack of experimental data. The FASER experiment is proposed to measure particles, such as neutrinos and hypothetical dark-sector particles, at the forward location of the $14 \mathrm{TeV}$ proton-proton collisions at the LHC. As it corresponds to $100-\mathrm{PeV}$ proton interactions in fixed target mode, a precise measurement by FASER would provide information relevant for PeV-scale cosmic rays. By studying three flavor neutrinos with the dedicated neutrino detector (FASER $v$ ), FASER will lead to a quantitative understanding of prompt neutrinos, which is an important background towards the astrophysical neutrino observation by neutrino telescopes such as IceCube. In particular, the electron and tau neutrinos have strong links with charmed hadron production. And, the FASER measurements may also shed light on the unresolved muon puzzle at the high energy. FASER is going to start taking data in 2022. We expect about 8000 numu, 1300 nue and 20 nutau CC interactions at the TeV energy scale during Run 3 of the LHC operation (2022-2024) with a 1.1 tons emulsion-based neutrino detector. We report here the overview and prospect of the FASER experiment in relation to the cosmic-ray physics, together with the first LHC neutrino candidates that we caught in the pilot run held in 2018.
\end{abstract}

$37^{\text {th }}$ International Cosmic Ray Conference (ICRC 2021)

July 12th - 23rd, 2021

Online - Berlin, Germany

\footnotetext{
${ }^{*}$ Presenter
} 


\section{Introduction}

Atmospheric leptons (muons, neutrinos) are created by air showers initiated from primary cosmic-rays from extrasolar sources. Following the hadron cascade production, some of the hadrons decay into leptons. The high energy components are in the forward direction with respect to the primary cosmic ray. The atmospheric leptons have been measured by many experiments, however, the recent studies are encountering puzzling results or lack of understanding. For example, in the estimation of mean atomic number of high energy primary cosmic-ray, a discrepancy between the number of muons and air shower profile has been reported [1]. If we trust the measurement of number of muons, the average atomic number of primary cosmic-ray is above iron, as shown in Figure 1. This sounds unnatural and it is recognized as a problem, which is called muon puzzle [2]. People doubt about the forward meson production, in particular pion/kaon ratio may be not well reproduced in the MC, which results in the excess of muons in data. For this we lack accelerator data at the proper energy range.
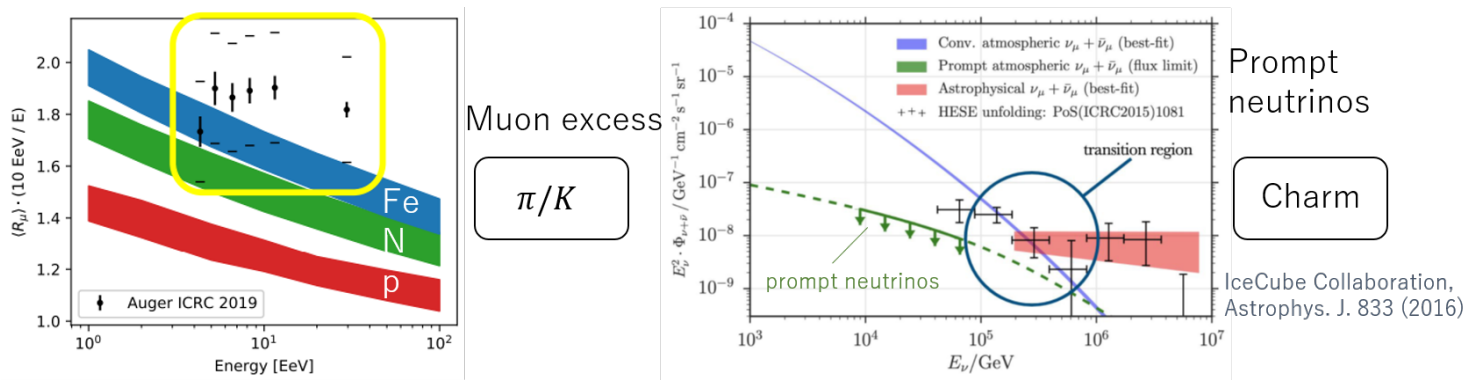

Figure 1: Muon excess problems and prompt neutrinos

Another example is the prompt atmospheric neutrino production. Prompt neutrinos are those from charmed hadron decays. As shown in Figure 1, it is expected to be a dominant background of astrophysical neutrino observations above $1 \mathrm{PeV}$ by neutrino telescopes, such as IceCube. Therefore it is important to understand their flux and energy spectra. However, there is currently no quantitative estimate of prompt neutrino production [3], namely forward charm meson production.

In summary, the understanding of forward particle production with flavor sensitivity such as $\pi$, $K$ and charm, is the key for future cosmic-ray experiments. This should be understood from beam experiments.

There are largely two kinds of experiments, fixed target experiments and collider experiments. Fixed target experiments like NA61 and NA65 can provide meson spectra with different target materials. However, they are limited in energy range. For example, $\sqrt{s}$ at the CERN SPS is only $\sim 30 \mathrm{GeV}$.

The collider method can go much higher. $\sqrt{s}$ is $14 \mathrm{TeV}$ at the LHC. This is equivalent to 100 $\mathrm{PeV}$ proton interaction in fixed target mode, covering above knee of cosmic ray flux, and also above the neutrino energy of interest. The data from the proton interactions at the LHC is at the proper energy range of interest. Yet, the conventional LHC experiments like ATLAS, CMS are not suitable because their angular acceptance is limited and they are not sensitive to forward emitted particles. Therefore, a dedicated forward detector is required. LHCf tried to measure neutral particles, namely $\gamma, \pi^{0}$ and $n$. That is still not enough to hit our questions related to flavors. 


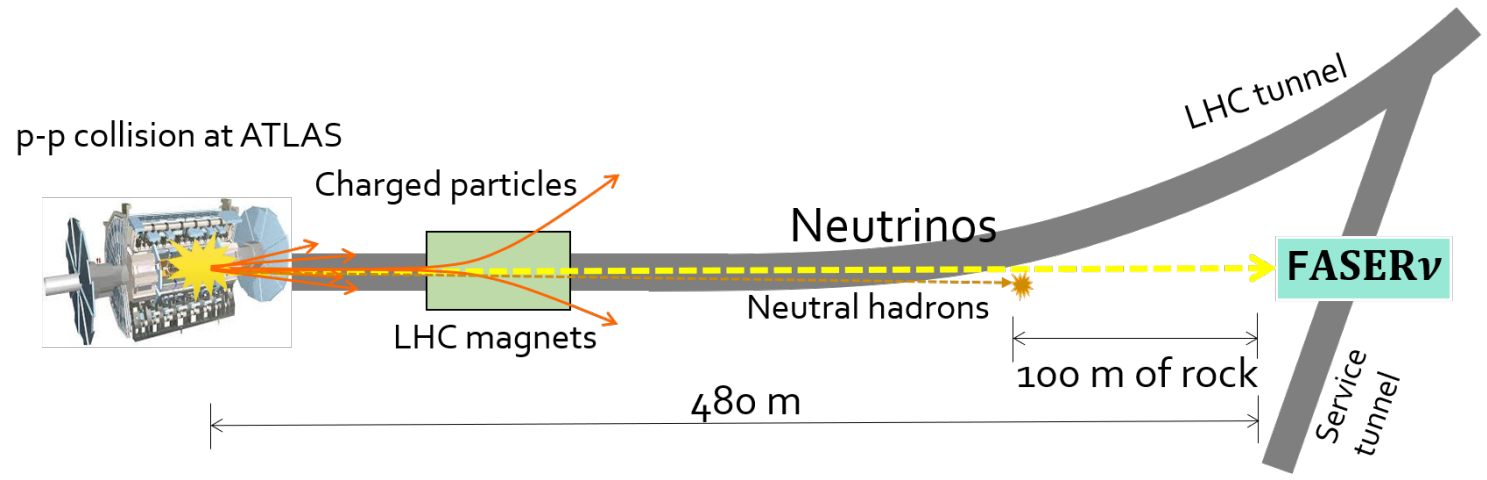

Figure 2: LHC's “Neutrino beamline" layout

FASER $v$ [4] comes here. FASER $v$ is going to study neutrinos from the LHC. As neutrinos at FASER $v$ have implications for different meson flavor productions, namely $\pi, K$ and charm particles as discussed in next section, FASER $v$ will provide important inputs to the hadron production models and to the cosmic-ray community.

\section{Neutrino beam at the $\mathrm{LHC}$}

Figure 2 shows the LHC's neutrino beamline. This was actually not designed as a neutrino beamline, but it functions as a high energy neutrino beamline. At the proton-proton interaction point, an intense beam of mesons is created in the forward direction. Some of them decay into neutrinos before the LHC magnet. Then, charged particles are deflected away by the magnets and neutral hadrons stop in the rock. Only neutrinos can go straight through $100 \mathrm{~m}$ of rock. By chance there is a disused service tunnel, where we will put FASER $v$ detector. In this way, we can exploit the intense neutrino beam from the LHC with a minimum cost for infrastructure. FASER $v$ is the first experiment using a collider as a neutrino source. FASER experiment [5], which is originally designed for new particle searches, and also FASER neutrino experiment $[4,6]$ were both approved by CERN in 2019.

Figure 3 shows the neutrino spectra at FASER $v$. We can get neutrinos at the TeV energy scale where there is no data available. We can measure neutrinos at the highest energy for $v_{e}$ and $v_{\tau}$. For $v_{\mu}$, we will fill the gap between the IceCube measurement and accelerator data. Using these neutrinos, we can study production, propagation and interactions of high energy neutrinos at the completely new kinematical regime. The physics potential in general is described in [4].

It is worth stressing that FASER $v$ can study different hadron flavor productions by measuring neutrinos energy spectra and neutrino flavors. Figure 4 shows the neutrino flux contributions from different hadrons [7]. For muon neutrinos, low energy part is due to $\pi$ decays, on the other hand, the high energy part is due to $K$ decays. Furthermore, their contributions depend on neutrino flavors. For electron neutrinos, $\pi$ contribution is negligible, but high energy part is made of charmed meson decays. Tau neutrinos are exclusively from charmed meson (mostly $D_{s}$ ) decays. Therefore, by measuring the neutrino energy spectrum, we can unfold the parental meson spectra. Therefore, FASER $v$ can provide important inputs to validate and improve hadron generators, eventually attacking muon puzzle and prompt neutrino production. 



Figure 3: Neutrino fluxes at the FASER $v$ and the projected cross section sensitivities in Run 3 (2022-2024) [4]. The short error bars are due to statistical uncertainty, the long are uncertainty due to the range of neutrino flux predictions (DPMJet is not considered).
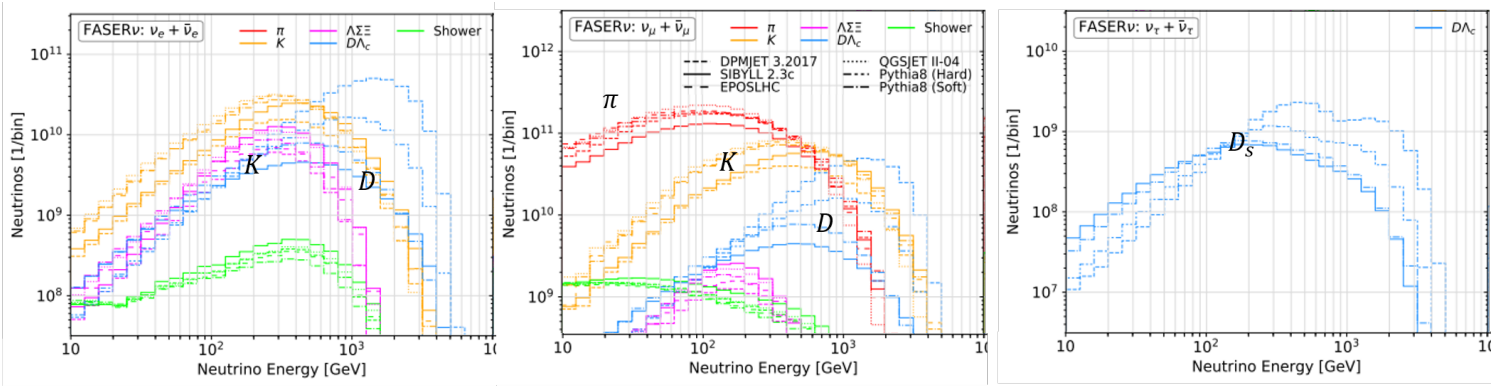

Figure 4: Neutrino fluxes, contributions from different mesons [7].

Furthermore, the neutrino measurements can provide deep insights on QCD. At the LHC energy, forward charm particles are created mostly by gluon-gluon interactions. Figure 5 shows the kinematical features of gluons which contribute to electron neutrino flux at FASER $v$ through charmed particles. Those are either large- $x$ or very small- $x$ below $10^{-5}$. Such a characteristic kinematical feature is ideal to test the small- $x$ factorization and also to test intrinsic charm. This is of interest of fundamental QCD processes, as well as important to understand prompt neutrinos.

\section{FASER $v$ detector and expected performance in Run 3}

The detector setup in Run 3 of LHC operation from 2022 to 2024 is shown in Figure 6. The FASER $v$ detector is made of emulsion tracker and tungsten target plates. It is followed by the FASER spectrometer. In the FASER $v$ detector, thanks to the high precision emulsion tracker, three neutrino flavors can be separated by their topology. Electron will quickly develop electromagnetic showers, muon will penetrate the detector, tau will make decay topology. The target mass of FASER $v$ is 1.1 tons. The energy resolution is estimated to be about $\Delta P / P \simeq 30 \%$. There is an interface detector, which allows to make a global reconstruction with the FASER spectrometer, making it possible to separate muon neutrino and anti-muon neutrinos.

It's a relatively small detector, however, we expect a lot of neutrino interactions of the order of $10^{4}$ in Run 3. This table shows the expected number of CC interactions in FASER $v$. Muon 


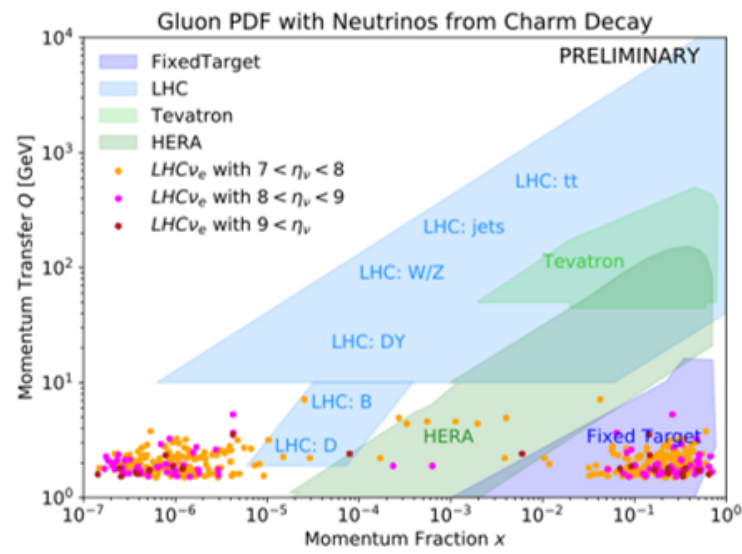

Figure 5: Gluon kinematical features for those contributing to electron neutrino fluxes at FASER $v$ through charmed particles.

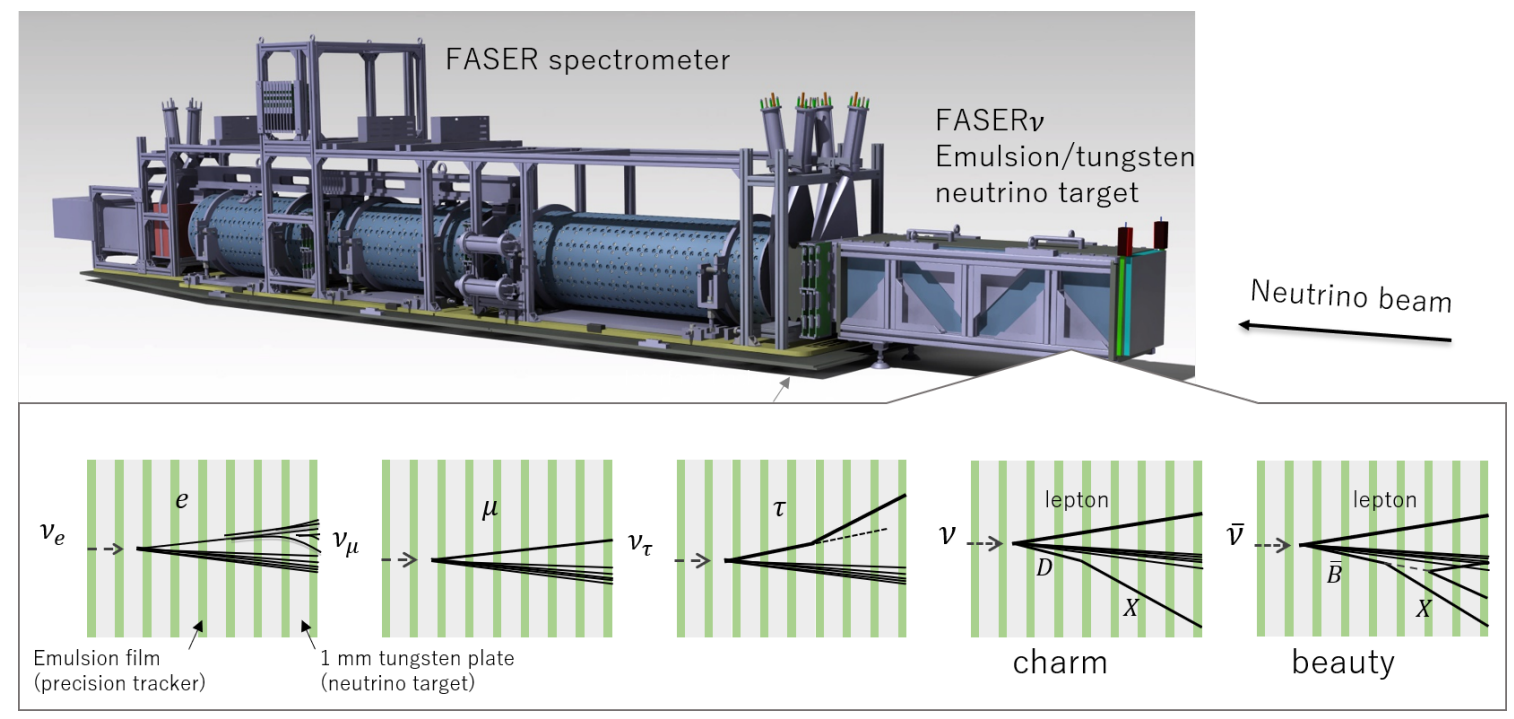

Figure 6: Detector configuration in Run 3

neutrinos are most abundant, but we will have electron neutrinos and even some tau neutrinos. The numbers largely depend on the hadron production models, meaning that FASER $v$ are sensitive to validate the hadron production models. The expected cross section sensitivities are shown in Figure 3 .

For the proof of principle, we already carried out a pilot run in 2018. Figure 7-left the photo of the $30 \mathrm{~kg}$ neutrino detector, which was exposed to the LHC's neutrino beam, collecting 12.2 $\mathrm{fb}^{-1}$ of data. The observed tracks in a small volume of $2 \mathrm{~mm} \times 2 \mathrm{~mm} \times 10$ emulsion films are shown here. There are a lot of tracks but they are found to be muons and electrons, so that they will not bother our neutrino measurements. After searching for neutrino interaction vertices, we found 18 candidates. Figure 8 shows the result of multivariate analysis, with signal (blue) and background (green) estimations, and data (black). The statistical significance from NULL hypothesis is calculated to be $2.7 \sigma$ [8]. This result demonstrates the detection of neutrino events 


\begin{tabular}{|c|c|c|c|}
\hline & SIBYLL & Pythia 8 & $\begin{array}{c}\text { DPMJET } \\
\text { (used in } \\
\text { FLUKA) }\end{array}$ \\
\hline$v_{e}, \bar{v}_{e}$ & 800,452 & 826,477 & 3390,1024 \\
\hline$v_{\mu}, \bar{v}_{\mu}$ & 6571,1653 & 7120,2178 & 8437,2737 \\
\hline$v_{\tau}, \bar{v}_{\tau}$ & 16,6 & 22,11 & 111,43 \\
\hline
\end{tabular}

Table 1: The number of expected neutrino events ant comparison among different hadron production generators.
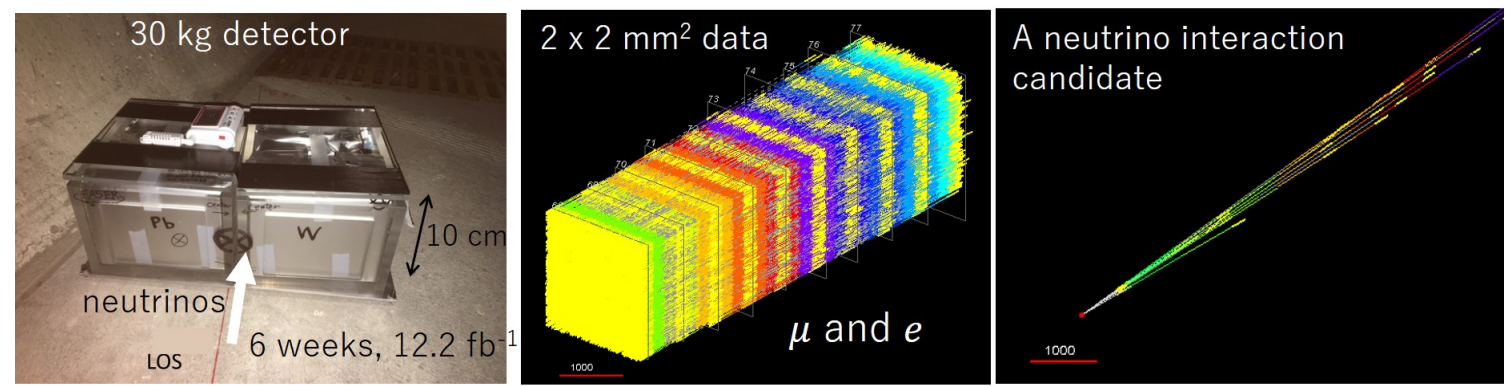

Figure 7: Left: A photo of pilot run detectors. Center: Reconstructed tracks in a small volume of detector. Right: A neutrino interaction candidate.

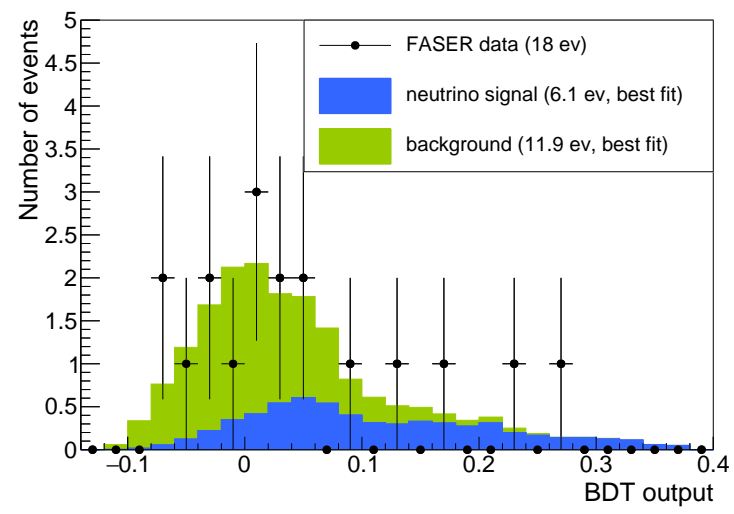

Figure 8: Output of the BDT (Boosted Decision Tree). The MC distributions are fitted to data.

at the actual LHC environment.

We are currently preparing for the physics run which is starting in 2022. Figure 9 shows some of photos from the activity. See the caption for the explanation. The FASER detector was installed in the LHC tunnel and under commissioning. The FASER $v$ detector structure is produced and its installation test was done (FASER $v$ is an replaceable module in order to exchange emulsion films.) The emulsion film production was tested and tungsten plates are being delivered. The FASER collaboration will make sure all the detector components are ready for data taking. 

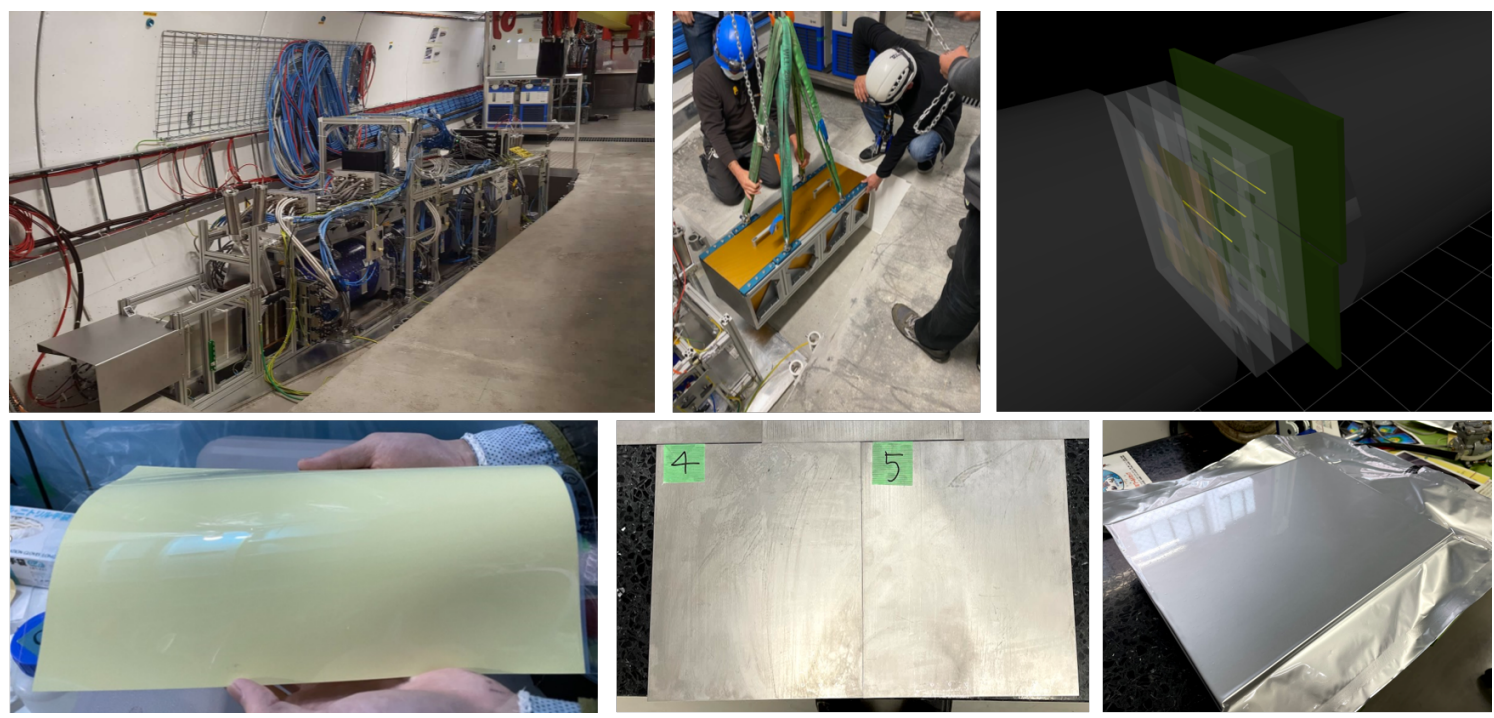

Figure 9: Preparation for the physics run starting in 2022. From top-left, the FASER detector installed in the LHC tunnel, the FASER $v$ detector installation test, a cosmic-ray track observed by the FASER's tracker at the LHC tunnel during the commissioning. From bottom-left, an emulsion film produced at the film production facility, tungsten plates, a sub-module of the emulsion/tungsten module.

\section{Conclusion}

FASER $v$ is the first experiment making use of neutrinos from a collider, which is approved and will start data taking in 2022. Thanks to its unique setup, we can study production, propagation and interactions of high energy neutrinos. In particular, it will provide vital inputs to the cosmic ray community in understanding of atmospheric lepton productions. The detection of neutrinos at the actual site was already demonstrated in 2018 samples. The FASER Collaboration is preparing the detector for the physics data taking in 2022 .

\section{Acknowledgments}

We thank CERN for the excellent performance of the LHC and the technical and administrative staff members at all FASER institutions. We also gratefully acknowledge invaluable assistance from many groups at CERN, particularly the Physics Beyond Colliders study group; the ATLAS Collaboration for providing the luminosity value; the NA65/DsTau Collaboration for providing their spare emulsion films and tungsten plates for this measurement, and Masahiro Komatsu for useful discussions. This work was supported in part by Heising-Simons Foundation Grant Nos. 2018-1135, 2019-1179, and 2020-1840, Simons Foundation Grant No. 623683, and the Department of Energy Grant No. DE-SC0016013. This work was supported by JSPS KAKENHI Grant Nos. JP19H01909, JP20H01919, JP20K04004, JP20K23373, a research grant from the Mitsubishi Foundation, and the joint research program of the Institute of Materials and Systems for Sustainability. 


\section{References}

[1] L. Cazon, Working Group Report on the Combined Analysis of Muon Density Measurements from Eight Air Shower Experiments, PoS ICRC2019 (2019) 214.

[2] A.A. Petrukhin, Muon puzzle in cosmic ray experiments and its possible solution, Nucl. Instrum. Meth. A 742 (2014) 228.

[3] M.G. Aartsen, K. Abraham, M. Ackermann, J. Adams, J.A. Aguilar, I. Ansseau et al., Observation and characterization of a cosmic muon neutrino flux from the northern hemisphere using six years of icecube data, Astrophysical Journal 833 (2016) .

[4] FASER collaboration, Detecting and Studying High-Energy Collider Neutrinos with FASER at the LHC, Eur. Phys. J. C 80 (2020) 61 [1908. 02310].

[5] FASER collaboration, Technical Proposal for FASER: ForwArd Search ExpeRiment at the LHC, 1812.09139.

[6] FASER collaboration, Technical Proposal: FASERnu, 2001.03073.

[7] F. Kling, Forward Neutrino Fluxes at the LHC, 2105.08270.

[8] FASER collaboration, First neutrino interaction candidates at the LHC, 2105. 06197. 


\section{Full Authors List: FASER Collaboration}

Akitaka Ariga ${ }^{*}, 1,2$, Henso Abreu ${ }^{3}$, Yoav Afik ${ }^{3}$, Claire Antel ${ }^{4}$, Tomoko Ariga ${ }^{5}$, Florian Bernlochner ${ }^{6}$, Tobias Boeckh ${ }^{6}$, Jamie Boyd $^{7}$, Lydia Brenner ${ }^{7}$, Franck Cadoux ${ }^{4}$, David W. Casper ${ }^{8}$, Charlotte Cavanagh $^{9}$, Xin Chen $^{10}$, Andrea Coccaro ${ }^{11}$, Monica D' Onofrio $^{9}$, Candan Dozen ${ }^{10}$, Yannick Favre ${ }^{4}$, Deion Fellers ${ }^{12}$, Jonathan L. Feng ${ }^{8}$, Didier Ferrere ${ }^{4}$, Stephen Gibson ${ }^{13}$, Sergio Gonzalez-Sevilla ${ }^{4}$, Carl Gwilliam ${ }^{9}$, Shih-Chieh $\mathrm{Hsu}^{14}$, Zhen $\mathrm{Hu}^{10}$, Giuseppe Iacobucci ${ }^{4}$, Tomohiro Inada ${ }^{10}$, Sune Jakobsen ${ }^{7}$, Enrique Kajomovitz ${ }^{3}$, Felix Kling ${ }^{15}$, Umut Kose ${ }^{7}$, Susanne Kuehn ${ }^{7}$, Helena Lefebvre ${ }^{13}$, Lorne Levinson ${ }^{16}$, $\mathrm{Ke} \mathrm{Li}^{14}$, Jinfeng Liu ${ }^{10}$, Chiara Magliocca ${ }^{4}$, JoshMcFayden $^{17}$, Sam Meehan ${ }^{7}$, Dimitar Mladenov ${ }^{7}$, Mitsuhiro Nakamura ${ }^{18}$, Toshiyuki Nakano ${ }^{18}$, Marzio Nessi ${ }^{7}$, Friedemann Neuhaus ${ }^{19}$, Laurie Nevay ${ }^{13}$, Hidetoshi Otono ${ }^{5}$, Carlo Pandini ${ }^{4}$, Hao Pang ${ }^{10}$, Lorenzo Paolozzi ${ }^{4}$, Brian Petersen ${ }^{7}$, Francesco Pietropaolo ${ }^{7}$, Markus Prim ${ }^{6}$, Michaela Queitsch-Maitland ${ }^{7}$, Filippo Resnati ${ }^{7}$, Hiroki Rokujo ${ }^{18}$, Jakob Salfeld-Nebgen ${ }^{7}$, Osamu Sato ${ }^{18}$, Paola Scampoli $^{1,20}$, Kristof Schmieden ${ }^{19}$, Matthias Schott ${ }^{19}$, Anna Sfyrla ${ }^{4}$, Savannah Shively ${ }^{8}$, John Spencer ${ }^{14}$, Yosuke Takubo ${ }^{21}$, Ondrej Theiner ${ }^{4}$, Eric Torrence ${ }^{12}$, Sebastian Trojanowski ${ }^{22}$, Serhan Tufanli ${ }^{7}$, Benedikt Vormwald ${ }^{7}$, Di Wang ${ }^{10}$, and Gang Zhan ${ }^{10}$

${ }^{1}$ Albert Einstein Center for Fundamental Physics, Laboratory for High Energy Physics, University of Bern, Sidlerstrasse 5, CH3012 Bern, Switzerland. ${ }^{2}$ Department of Physics, Chiba University, 1-33 Yayoi-cho Inage-ku, Chiba, 263-8522, Japan. ${ }^{3}$ Department of Physics and Astronomy, Technion-Israel Institute of Technology, Haifa 32000, Israel. ${ }^{4}$ Département de Physique Nucléaire et Corpusculaire, University of Geneva, CH-1211 Geneva 4, Switzerland. ${ }^{5}$ Kyushu University, Nishi-ku, 819-0395 Fukuoka, Japan. ${ }^{6}$ Universität Bonn, Regina-Pacis-Weg 3, D-53113 Bonn, Germany. ${ }^{7}$ CERN, CH-1211 Geneva 23, Switzerland. ${ }^{8}$ Department of Physics and Astronomy, University of California, Irvine, CA 92697-4575, USA. ${ }^{9}$ University of Liverpool, Liverpool L69 3BX, United Kingdom. ${ }^{10}$ Department of Physics, Tsinghua University, Beijing, China. ${ }^{11}$ INFN Sezione di Genova, Via Dodecaneso, 33-16146, Genova, Italy. ${ }^{12}$ University of Oregon, Eugene, OR 97403, USA. ${ }^{13}$ Royal Holloway, University of London, Egham, TW20 0EX, UK. ${ }^{14}$ Department of Physics, University of Washington, PO Box 351560, Seattle, WA 98195-1560, USA. ${ }^{15}$ SLAC National Accelerator Laboratory, 2575 Sand Hill Road, Menlo Park, CA 94025, USA. ${ }^{16}$ Department of Particle Physics and Astrophysics, Weizmann Institute of Science, Rehovot 76100, Israel. ${ }^{17}$ Department of Physics \& Astronomy, University of Sussex, Sussex House, Falmer, Brighton, BN1 9RH, United Kingdom. ${ }^{18}$ Nagoya University, Furo-cho, Chikusa-ku, Nagoya 464-8602, Japan. ${ }^{19}$ Institut für Physik, Universität Mainz, Mainz, Germany. ${ }^{20}$ Dipartimento di Fisica "Ettore Pancini", Università di Napoli Federico II, Complesso Universitario di Monte S. Angelo, I-80126 Napoli, Italy. ${ }^{21}$ Institute of Particle and Nuclear Study, KEK, Oho 1-1, Tsukuba, Ibaraki 305-0801, Japan. ${ }^{22}$ Astrocent, Nicolaus Copernicus Astronomical Center Polish Academy of Sciences, ul. Bartycka 18, 00-716 Warsaw, Poland. 